\title{
Kinetostatics and Dynamics of Redundantly Actuated Planar Parallel Link Mechanisms
}

\author{
Takashi Harada \\ Kinki University \\ Japan
}

\section{Introduction}

Robotic systems with parallel link mechanisms (PLMs) have mechanical characteristics such as rigidity of the mechanism and precise positioning (Stewart, 1966), (Merlet 2006), (Wang \& Liu, 2008). These characteristics enable them to stably perform contact tasks with sensitive force, e.g. mold grindings and rehabilitation robotics. On the other hand, mechanical interference and the singularity of the mechanisms (Merlet, 1989) restrict the robot's movable range. PLMs have therefore been conventionally applied not to general-purpose industrial robots, but to special-purpose machines (Weck, 2002), (Oiwa, 1997).

In order to expand this limited application of PLMs, we have proposed a new parallel link mechanism with multi drive linear motors (MDLMs) (Harada \& Nagase, 2009, 2010). The multi drive is a control method for linear motors in which a number of moving parts are individually driven on one stator part. We have proposed various configurations of PLMs which have been constructed for MDLMs. These PLMs expand the robot's movable range while retaining the advantageous rigid mechanism and precise positioning that PLMs offer. Moreover, the proposed PLMs are suitable for force control, because the linear motors are directory driven without friction full gearings.

Several studies related to expanding the movable range of the PLM have previously been published (Honegger et al., 1997), (Kim et al., 2003), (Liu et al., 2004), (In et al., 2008), (Milutinovis et al., 2005), (Zhang, 2008). Notably, redundantly actuated 3-DOF $x y \theta$ planar PLMs on linear actuators (Zhang, 2008), (Wang et al., 2008), (Marquet et al., 2001) have been proposed as mechanisms that are similar to our PLM. A two 2-DOF PRRRP (P denotes prismatic joint and $\mathrm{R}$ denotes rotational) parallel manipulator (Liu et al., 2007) has been employed as a mechanical element of these planar PLMs, including ours. However, these planar PLMs, excluding ours, aim at position control, not at force control. Conventionally, gearings or ball screws are used for the actuator transmission of PLMs. However, it is difficult to compensate for the undesired internal force among the redundant link mechanisms with the position controlled actuators. This undesired internal force results in mechanical deformation around the transmission parts (Leong et al., 2004).

Our PLM is suitable for force control, because it employs directly driven linear motors. It can control the internal force and compensate for the mechanical deformation because of the favorable effect of force control and the back-drivability of the directly driven linear motors. 
Moreover, it is designed to have constant inertia and decoupled dynamics in the $x$ (horizontal) direction. Gravitational force only affects it in the $y$ (vertical) direction. The simple characteristics of the dynamics make it easy to install a fast acceleration control or impedance control (Hogan, 1985) to the PLM, where the nonlinear dynamics of the PLM must be compensated for.

In this paper, we investigate the kinetostatics (kinametics and static force), and dynamics characteristics of the 3D4M PLM using symbolic mathematical analysis and numerical simulations. This paper is organized as follows: first, configurations of the 3D4M PLM on multi drive linear motors are introduced. Second, kinematic equations, forward kinematics and derivative kinematics of the 3D4M PLM are derived. The derived equations are symbolically programmed using Mathematica. Next, singularity and static forces of the 3D4M PLM are analyzed using Mathematica. Then, the decoupled dynamical design of the 3D4M PLM is introduced. The equations of motion of the 3D4M PLM are derived by symbolic programming using Mathematica. Finally, some examples are introduced for solving the equations of motion numerically using Mathematica.

\section{Configurations of link mechanism}

\subsection{Multi drive linear motor}

A ball screw driven by a rotational motor, as shown in Fig.1 (a), is generally used as a linear actuator in conventional PLMs. A single driving part moves in a straight line on a linear stator; we will refer to this below as a single drive. The single drive disturbs the space in which movement takes place, and restricts the general-purpose application of PLMs. Moreover, it is difficult in principal for the load of the tip to be transmitted back to the actuator, which in turn renders the ball screw drive incapable of force control.

To cope with these problems, multi drive linear motors (MDLMs), as shown in Fig.1 (b), are employed in our research. MDLMs offer a way to arrange more than one moving parts on one stator of a linear motor, with each moving part individually controlled and driven.

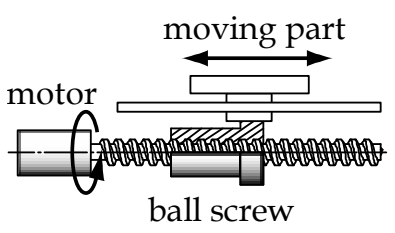

(a) single drive

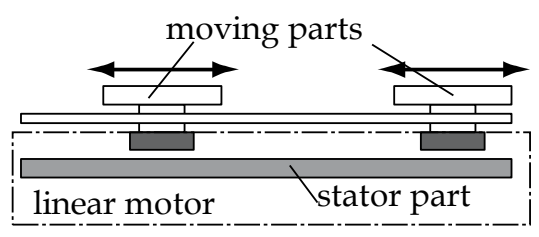

(b) multi drive

Fig. 1. Single and multi drive linear motors

\subsection{Configuration of 3-DOF planar mechanisms}

Configurations of a 3-DOF $(x y \theta)$ planar PLM with 3 non-redundant moving parts (3D3M) and a 3-DOF $(x y \theta)$ planar PLM with 4 redundant moving parts $(4 \mathrm{D} 4 \mathrm{M})$, are shown in Figs. 2(a) and (b). The 3D4M PLM with 4 redundant moving parts is the centerpiece of our research. The redundancy of the PLMs is not used only for singularity avoidance as sought by conventional research, but is also used for forward kinematics computation (Merlet, 1996) and calibration of the mechanism (Zhuang \& Liu, 1998), (Chiu \& Perg, 2003), which have been standing problems with conventional PLMs. 
We are planning to apply the PLM to a table mechanism of 5 axis machine tools. A schematic view and the motions of the 3D4M PLM are illustrated in Fig. 3 and Fig. 4.

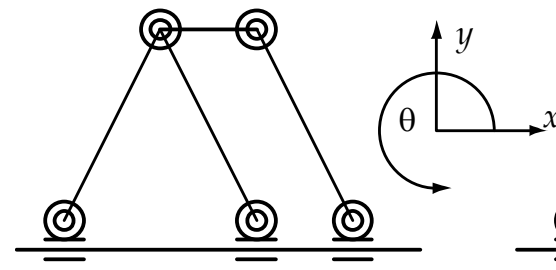

(a) 3D3M

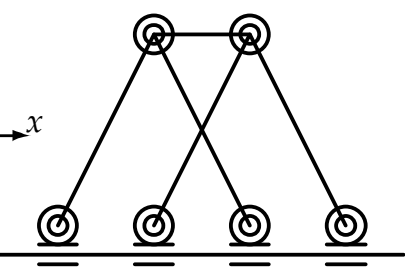

(b) 3D4M

Fig. 2. 3-DOF planar parallel mechanisms

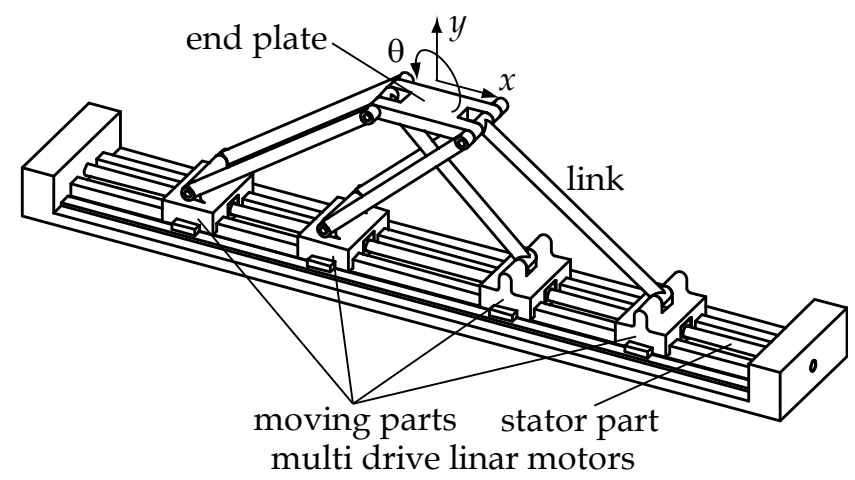

Fig. 3. Schematic view of the 3D4M mechanism

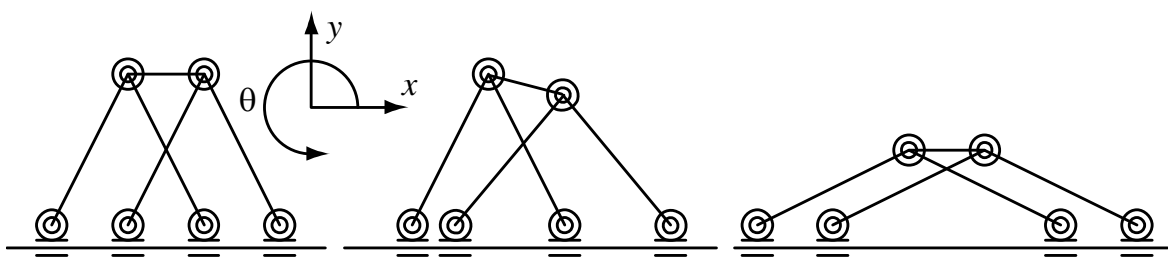

Fig. 4. Motions of the 3D4M mechanism

\section{Kinematics of the parallel link mechanism}

\subsection{Kinematics of 3-DOF parallel link mechanisms}

On the basis of the general kinematics formulation of parallel link mechanisms (Arai et al., 1991), kinematics equations for the proposed 3-DOF parallel link mechanism are derived. In turn, the parallel link mechanism of the particular configuration of our research can also be analyzed by the general method of kinematics.

The kinematic relationships of the 3D4M PLM, as shown in Fig. 5, are expressed as follows: 


$$
\begin{aligned}
& \mathbf{p}+\mathbf{R s}_{i}=\mathbf{L}_{i}=c_{i} \mathbf{a}+l_{i} \mathbf{z}_{i} \\
& \mathbf{p}=[x, y]^{T} \\
& \mathbf{a}=[1,0]^{T} \\
& \mathbf{R}=\left[\begin{array}{cc}
\cos \theta & -\sin \theta \\
\sin \theta & \cos \theta
\end{array}\right] . \\
& \mathbf{s}_{1}=\mathbf{s}_{2}=\left[-r_{t}, 0\right]^{T} \\
& \mathbf{s}_{3}=\mathbf{s}_{4}=\left[r_{t}, 0\right]^{T}
\end{aligned}
$$

where $c_{i}(i=1, . ., 4)$ is the control variable of the $i$ th actuator, i.e., the position of the $i$ th moving part of the multi drive linear motor. The length of the $i$ th rod (link) is expressed as $l_{i}$. The distance from the central point of the end plate to the $i$ th pair of the end plate is expressed as $r_{t}$. Other symbols are indicated as in Fig. 5 .

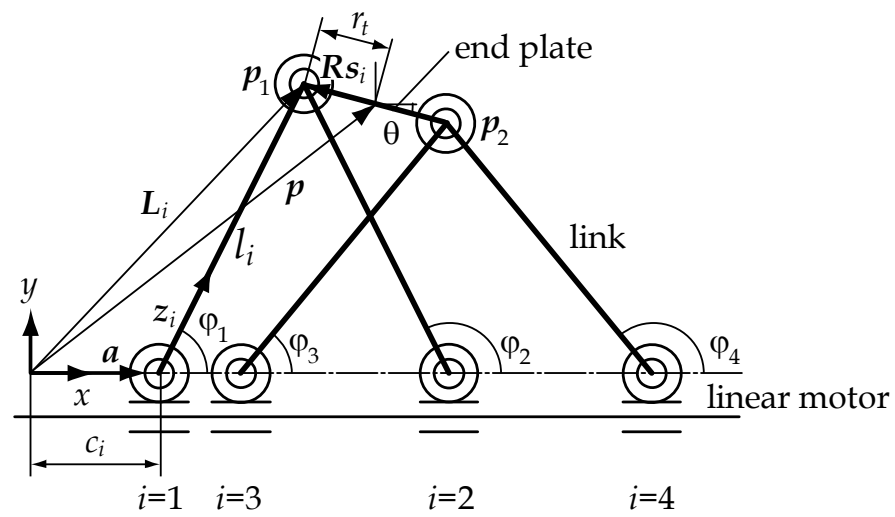

Fig. 5. Kinematic model of the 3-DOF PLM

Equation (1) expresses the relationship between the positions of the moving parts $c_{i}(i=1, . ., 4)$ and the positions $\mathbf{p}$ and orientation $\theta$ of the end plate. By solving (1) as $c_{i}$, an inverse kinematics equation is derived as follows:

$$
c_{i}=\mathbf{L}_{i}^{T} \mathbf{a} \pm \sqrt{\left(\mathbf{L}_{i}^{T} \mathbf{a}\right)^{2}-\left(\mathbf{L}_{i}^{T} \mathbf{L}_{i}\right)^{2}+l_{i}^{2}} .
$$

For the 1st and 3rd link, the plus-minus sign in (2) is given as positive, and for 2nd and 4 th link is given as negative. The unit direction vector $\mathbf{z}_{i}$ and angle $\varphi_{i}$ of the $i$ th rod are given as

$$
\begin{aligned}
& \mathbf{z}_{i}=\left[z_{x i}, z_{y i}\right]^{T}=\left(\mathbf{L}_{i}-c_{i} \mathbf{a}\right) / l_{i} \\
& \varphi_{i}=\tan ^{-1}\left(\frac{z_{y i}}{z_{x i}}\right)
\end{aligned}
$$

By applying derivatives to both sides of (1), the derivative relation of the 3D4M PLM is derived as follows: 


$$
\begin{aligned}
& \mathbf{J}_{e 43} \Delta \mathbf{p}_{3}=\mathbf{J}_{c 4} \Delta \mathbf{c}_{4} \\
& \Delta \mathbf{p}_{3}=[\Delta x, \Delta y, \Delta \theta]^{T} \\
& \Delta \mathbf{c}_{4}=\left[\Delta c_{1}, \ldots, \Delta c_{4}\right]^{T} \\
& \mathbf{J}_{e 43}=\left[\begin{array}{c|c}
\mathbf{z}_{1}{ }^{T} & \mathbf{z}_{1}{ }^{T}\left(\mathbf{R}_{\theta} \mathbf{R s}_{1}\right) \\
\vdots & \vdots \\
\mathbf{z}_{4}{ }^{T} & \mathbf{z}_{4}{ }^{T}\left(\mathbf{R}_{\theta} \mathbf{R s}_{4}\right)
\end{array}\right], \mathbf{J}_{c 4}=\left[\begin{array}{ccc}
\mathbf{z}_{1}{ }^{T} \mathbf{a} & \cdots & 0 \\
\vdots & \ddots & \vdots \\
0 & \cdots & \mathbf{z}_{4}{ }^{T} \mathbf{a}
\end{array}\right] \\
& \mathbf{R}_{\theta}=\left[\begin{array}{cc}
0 & -1 \\
1 & 0
\end{array}\right]
\end{aligned}
$$

Where $\mathbf{J}_{e 43}$ and $\mathbf{J}_{c 4}$ are Jacobian matrices of the system. Kinematic characteristics such as singular point and static force can be analyzed by using Eq. (4).

The kinematic equation of the 3D3M PLM is derived by removing the redundant part from Eqs. (1)-(4) as follows:

$$
\begin{aligned}
& \mathbf{J}_{e 33} \Delta \mathbf{p}_{3}=\mathbf{J}_{c 3} \Delta \mathbf{c}_{3} \\
& \Delta \mathbf{p}_{3}=[\Delta x, \Delta y, \Delta \theta]^{T} . \\
& \Delta \mathbf{c}_{3}=\left[\Delta c_{1}, \Delta c_{2}, \Delta c_{4}\right]^{T} .
\end{aligned}
$$

\subsection{Symbolical programming using Mathematica}

Equations (1)-(5) are symbolically programmed using Mathematica. Mathematica is a computational software program widely used in scientific, engineering, and mathematical fields and other areas of technical computing.

Positional vector $\mathbf{p}$ and matrix $\mathbf{R}$ in Eq. (1) are symbolically defined by Mathematica.

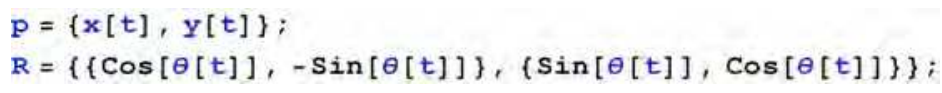

Program code of Mathematica is indicated as (M. i). Valuables $x[t], y[t]$ and $\theta[t]$ in (M. 1) are defined as function of time $t$.

Vectors $\mathbf{s}_{i}, \mathbf{L}_{i}(i=1, \ldots 4)$ and $\mathbf{a}$ in Eq. (1) are defined as

$$
\begin{aligned}
& \text { s1 }=\{-r t, 0\} ; \\
& \text { s2 }=\{-r t, 0\} ; \\
& \text { s3 }=\{r t, 0\} ; \\
& \text { s4 }=\{r t, 0\} ; \\
& \text { L1 }=p+R . s 1 ; \\
& \text { L2 }=p+R . s 2 ; \\
& \text { L3 }=p+R . s 3 ; \\
& \text { L4 }=p+R . s 4 ; \\
& a=\{1,0\} ;
\end{aligned}
$$

The position of each moving part $c_{i}(i=1, \ldots, 4)$ in Eq. (2) is expressed as 


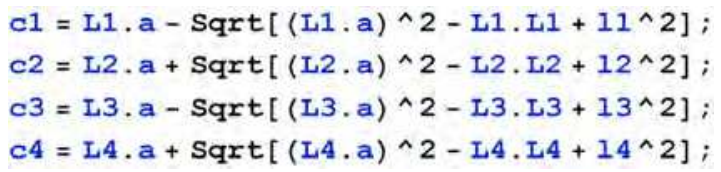

The unit direction vector $\mathbf{z}_{i}$ and angle $\varphi_{i}(i=1, \ldots, 4)$ of the $i$ th rod in Eq. (3) are defined as

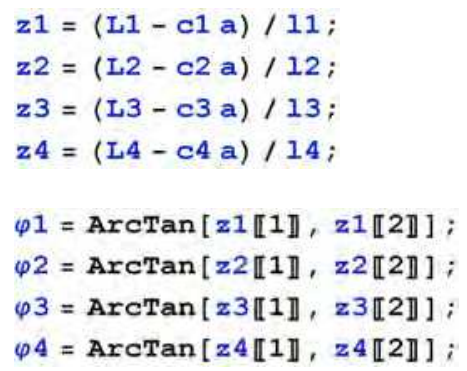

Matrix $\mathbf{R}_{\theta}$ in Eq. (4) is defined as

$$
R \theta=\{\{0,-1\},\{1,0\}\} \text {;. }
$$

Jacobian Matrices $\mathbf{J}_{e 43}$ in Eq. (4) and $\mathbf{J}_{e 33}$ in Eq. (5) are expressed as

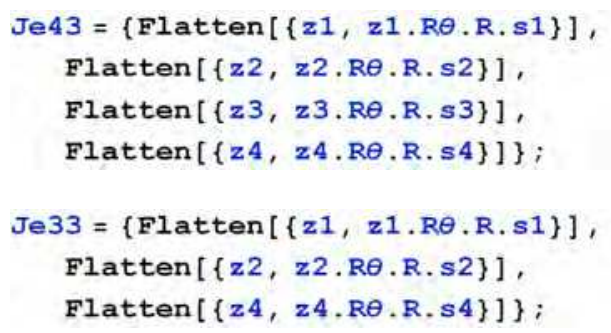

\subsection{Forward kinematics}

As shown in Fig. 5, two links are connected to the same pair of each end of the end plate. This makes it easy to solve the forward kinematics solution. The positions of the kinematic pairs $p_{1}:\left[x_{1}, y_{1}\right]^{T}$ and $p_{2}:\left[x_{2}, y_{2}\right]^{T}$ are expressed by the $c_{i}$, the position of the moving part of the linear motor, as follows:

$$
\begin{aligned}
& {\left[x_{1}, y_{1}\right]^{T}=\left[c_{1}+l_{1} \cos \varphi_{1}, l_{1} \sin \varphi_{1}\right]^{T}} \\
& \varphi_{1}=\cos ^{-1}\left(\frac{l_{1}^{2}+\left(c_{2}-c_{1}\right)^{2}-l_{2}^{2}}{2 l_{1}\left(c_{2}-c_{1}\right)}\right) \\
& {\left[x_{2}, y_{2}\right]^{T}=\left[c_{3}+l_{3} \cos \varphi_{2}, l_{4} \sin \varphi_{2}\right]^{T}} \\
& \varphi_{2}=\cos ^{-1}\left(\frac{l_{3}^{2}+\left(c_{4}-c_{3}\right)^{2}-l_{4}^{2}}{2 l_{1}\left(c_{4}-c_{3}\right)}\right)
\end{aligned}
$$


If the length of each link $l_{i}$ is designed with the same value as $l$, the forward kinematics of the PLM becomes a simple formula, as follows:

$$
\begin{aligned}
& {\left[x_{1}, y_{1}\right]^{T}=\left[\left(c_{2}+c_{1}\right) / 2, \sqrt{l^{2}-\left(\left(c_{2}-c_{1}\right) / 2\right)^{2}}\right]^{T}} \\
& {\left[x_{2}, y_{2}\right]^{T}=\left[\left(c_{3}+c_{4}\right) / 2, \sqrt{l^{2}-\left(\left(c_{4}-c_{3}\right) / 2\right)^{2}}\right]^{T} .}
\end{aligned}
$$

The orientation and the central position of the end plate are given from the positions of the actuators $c_{i}$, as follows:

$$
\begin{aligned}
& x=\left(x_{1}+x_{2}\right) / 2 \\
& y=\left(y_{1}+y_{2}\right) / 2 \\
& \theta=\tan ^{-1}\left(\frac{y_{2}-y_{1}}{x_{2}-x_{1}}\right)
\end{aligned} .
$$

\subsection{Singularity of the 3-DOF PLM}

It is well known that parallel link mechanisms have two kinds of singularities (Gosselin \& Angels, 1990). When PLM arrives at a position and orientation such that the Jacobian matrices $\mathbf{J}_{c m}$ in Eqs. (4)-(5) are singular, the output of the actuator does not transfer to the link mechanism. These situations are referred to as the 1st kind of singularity. When the PLM arrives at a position and orientation such that the Jacobian matrices $\mathbf{J}_{e n}$ in Eqs. (4)-(5) are singular, the output of the end effecter does not transfer to the link mechanism. These situations are referred to as the 2nd kind of singularity. Variables $m$ and $n$ express the number of actuators and the degree of freedom of the end plate, respectively.

When each element of $\mathbf{J}_{c m}$ equals zero, the proposed PLM becomes the 1st kind of singularity. The condition of the 1st kind of singularity is expressed as follows:

$$
\mathbf{z}_{i}^{T} \mathbf{a}=0 .
$$

Equation (9) implies that if the unit direction vector a of the actuator and the unit direction vector $\mathbf{z}_{i}$ of each link are orthogonal, the PLM becomes one of the 1st kind of singular points. The 2nd kind of singularity differs, depending on the configuration of the PLM. Here, the singular points of the 3D3M PLM are derived. The conditions of the 2nd kind of singularities are given as the determinant of the Jacobian matrix in Eq. (5), which equals zero as follows:

$$
\operatorname{det}\left(\mathbf{J}_{e 33}\right)=0 \text {. }
$$

Using symbolic mathematics software, equation (10) is solved. In case of each rod length $l_{i}$ is identical as $l$, the following 4 types of singular points exist in the 3D3M PLM.

Type 1:

$$
\theta=\sin ^{-1}\left(y / r_{t}\right)
$$

Type 2:

$$
\theta=\sin ^{-1}\left(( \pm l+y) / r_{t}\right)
$$

Type 3:

$$
\theta= \pm \cos ^{-1}\left(\sqrt{\left(\left(l-r_{t}\right)^{2}-y^{2}\right) /\left(l-r_{t}\right)^{2}}\right.
$$


Type 4:

$$
\theta= \pm \cos ^{-1}\left(\sqrt{\left(\left(l+r_{t}\right)^{2}-y^{2}\right) /\left(l+r_{t}\right)^{2}}\right.
$$

\subsection{Singularity analysis using Mathmatica}

Here, we show how to derive the singularity conditions of Eqs. (11. 1) - (11. 4) using Mathematica.

When each rod length $l_{i}$ is identical, Jacobian Matrix $\mathbf{J}_{e 33}$ of $(\mathrm{M} .10)$ is redefined $\mathbf{J}_{e 33 a}$ as follows:

$$
\mathrm{Je} 33 \mathrm{a}=\mathrm{Je} 33 / . \quad\{11 \rightarrow 1,12 \rightarrow 1,13 \rightarrow 1,14 \rightarrow 1\} ; .
$$

At that time, $\operatorname{det}\left(\mathbf{J}_{e 33}\right)$ is simplified as follows:

Det [Je33a] // Simplify

$$
\begin{aligned}
& -\frac{1}{1^{3}} 2 r t(r t \sin [\theta[t]]-y[t]) \sqrt{1^{2}-(-r t \sin [\theta[t]]+y[t])^{2}} \\
& \quad\left(r t \sin [2 \theta[t]]+2 \cos [\theta[t]] y[t]+2 \sin [\theta[t]] \sqrt{1^{2}-(r t \sin [\theta[t]]+y[t])^{2}}\right) .
\end{aligned}
$$

(M. 13) is the output of (M. 12) by Mathematica. Equation (10) is solved by Mathematica as follows:

$$
\begin{aligned}
& \text { Solve[Det [Je33a] }=0, \theta[t]] / / \operatorname{Simplify} \\
& \left\{\left\{\theta[t] \rightarrow-\operatorname{ArcCOs}\left[-\frac{\sqrt{(1-r t)^{2}-y[t]^{2}}}{\sqrt{(1-r t)^{2}}}\right]\right\},\left\{\theta[t] \rightarrow \operatorname{ArcCos}\left[-\frac{\sqrt{(1-r t)^{2}-y[t]^{2}}}{\sqrt{(1-r t)^{2}}}\right]\right\},\right. \\
& \left\{\theta[t] \rightarrow-\operatorname{ArcCos}\left[\frac{\sqrt{(1-r t)^{2}-y[t]^{2}}}{\sqrt{(1-r t)^{2}}}\right]\right\},\left\{\theta[t] \rightarrow \operatorname{ArcCos}\left[\frac{\sqrt{(1-r t)^{2}-y[t]^{2}}}{\sqrt{(1-r t)^{2}}}\right]\right\}, \\
& \left\{\theta[t] \rightarrow-\operatorname{ArcCos}\left[-\frac{\sqrt{(1+r L)^{2}-y[L]^{2}}}{\sqrt{(1+r t)^{2}}}\right]\right\},\left\{\theta[t] \rightarrow \operatorname{ArcCos}\left[-\frac{\sqrt{(1+r L)^{2}-y[L]^{2}}}{\sqrt{(1+r t)^{2}}}\right]\right\}, \\
& \left\{\theta[t] \rightarrow-\operatorname{ArcCos}\left[\frac{\sqrt{(1+r t)^{2}-y[t]^{2}}}{\sqrt{(1+r t)^{2}}}\right]\right\},\left\{\theta[t] \rightarrow \operatorname{ArcCos}\left[\frac{\sqrt{(1+r t)^{2}-y[t]^{2}}}{\sqrt{(1+r t)^{2}}}\right]\right\}, \\
& \left.\left\{\theta[t] \rightarrow \operatorname{ArcSin}\left[\frac{y[t]}{r t}\right]\right\},\left\{\theta[t] \rightarrow \operatorname{ArcSin}\left[\frac{-1+y[t]}{r t}\right]\right\},\left\{\theta[t] \rightarrow \operatorname{ArcSin}\left[\frac{1+y[t]}{r t}\right]\right\}\right\}
\end{aligned}
$$

(M. 15) is the output of (M. 14) by Mathematica. Singular points of Eqs. (11.1) - (11. 4) are given by (M. 15).

\subsection{Singularity avoidance by redundantly actuation}

The singularity avoidance of the 3D4M PLM is shown through the definition of manipulability (Yoshikawa, 1985). The derivative kinematics of the PLM is transformed as

$$
\Delta \mathbf{c}_{3}=\mathbf{J}_{c 3}{ }^{-1} \mathbf{J}_{e 33} \Delta \mathbf{p}_{n}=\mathbf{J}_{c e 33} \Delta \mathbf{p}_{n} .
$$

The manipulability of the PLM is defined as follows: 


$$
w=\sqrt{\operatorname{det}\left(\mathbf{J}_{c e 33}^{T} \mathbf{J}_{c e 33}\right)} \text {. }
$$

Numerical calculations of the manipulability of the 3D3M PLM and the 3D4M PLM in correspondence with the rotation angle of the end plate, are shown in Figs. 6(a) and (b). The ratio of each link length $l_{i}$ and the length of the end effecter $2 r_{t}$ is given as 2:1.

The 3D3M non-redundant PLM becomes the 4th type of singular point given in (11.4) when the angle of the end plate equals 28.7 degrees. Around this angle, the manipulability becomes to zero, as shown in Fig.6 (a). On the other hand, the singular point is avoided by the redundant 3D4M PLM, as shown in Fig.6 (b). This confirms that the redundancy of the 3D4M PLM greatly increases homogeneous manipulability.

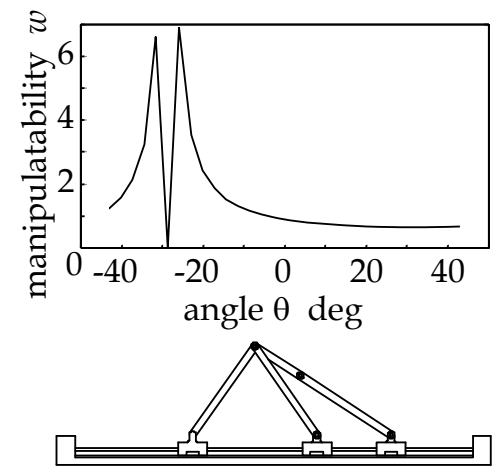

(a) 3D3M PLM
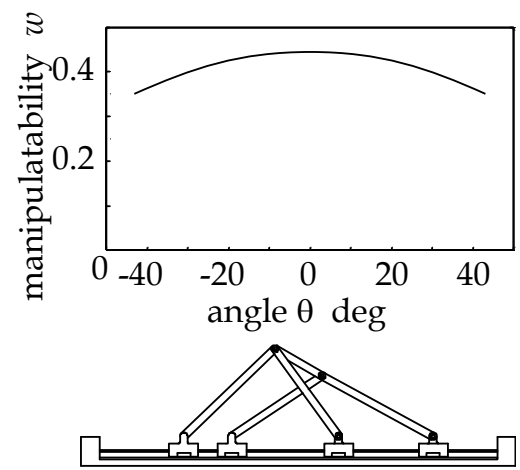

(b) 3D4M PLM

Fig. 6. Singularity analysis of the parallel link mechanisms

\section{Static force analysis of the PLM}

\subsection{Static force analysis for the non-redundant PLM}

Conventional static force analysis only derives the relationship between the generative force and torque of actuators and the external forces of the end effecter based on the principle of virtual force. Here, we expand this static force analysis in order to also calculate internal forces such as the constraint forces at the joints and the axial forces of the links.

First, the formula for static force is derived when the degree of freedom of the end effecter $(n)$ and the actuators $(m)$ is equivalent. For the sake of convenience, the external forces of the end plate $\mathbf{f}_{e}$ and the generative forces of the actuators $\mathbf{f}_{c}$ are expressed by vector forms as follows:

$$
\begin{aligned}
& \mathbf{f}_{e}=\left[f_{x}, f_{y}, \tau_{\theta}\right]^{T} \\
& \mathbf{f}_{c}=\left[f_{c 1}, \ldots, f_{c m}\right]^{T} .
\end{aligned}
$$

The relationship between $\mathbf{f}_{e}$ and $\mathbf{f}_{c}$ is derived by the principle of virtual forces as

$$
\begin{aligned}
\mathbf{f}_{e} & =\mathbf{J}_{c e n m}{ }^{T} \mathbf{f}_{c} \\
\mathbf{f}_{c} & =\left(\mathbf{J}_{c e n m}{ }^{T}\right)^{-1} \mathbf{f}_{e} .
\end{aligned}
$$


As shown in Fig. 7, $f_{n i}$ and $f_{l i}$ are defined as quantities of the constraint force at the $i$ th joint and axial force of the $i$ th link, respectively. The values $f_{n i}$ and $f_{l i}$ represent the internal forces of the PLM. The unit direction vector $\mathbf{n}$ along the constraint force $f_{n i}$ is orthogonal to the unit direction vector a along the actuator force $f_{c i}$. The equilibrium of forces at the $i$ th joint is given as:

$$
f_{c i} \mathbf{a}+f_{n i} \mathbf{n}+f_{l i} \mathbf{z}_{i}=\mathbf{0} .
$$

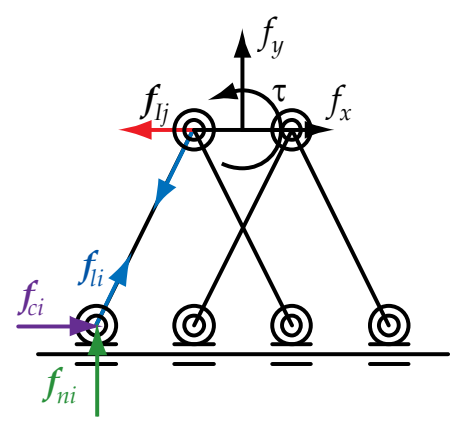

Fig. 7. Internal and external forces of the PLM

By applying the inner product to Eq. (16) with each vector $\mathbf{a}$ and $\mathbf{n}$, with the condition that vectors $\mathbf{a}$ and $\mathbf{n}$ are mutually orthogonal, the following formulas are derived.

$$
\begin{aligned}
& f_{c i}+\left(\mathbf{z}_{i}{ }^{T} \mathbf{a}\right) f_{l i}=0 \\
& f_{n i}+\left(\mathbf{z}_{i}{ }^{T} \mathbf{n}\right) f_{l i}=0
\end{aligned}
$$

The constraint force $f_{n i}$ and the axial force $f_{l i}$ of each link are combined as vector form $\mathbf{f}_{n}$ and $\mathbf{f}_{I}$ as follows:

$$
\begin{aligned}
& \mathbf{f}_{c}=-\operatorname{diag}\left(\mathbf{z}_{1}{ }^{T} \mathbf{a}, \ldots, \mathbf{z}_{m}{ }^{T} \mathbf{a}\right) \cdot \mathbf{f}_{l}=-\mathbf{J}_{c m} \mathbf{f}_{l} \\
& \mathbf{f}_{n}=-\operatorname{diag}\left(\mathbf{z}_{1}{ }^{T} \mathbf{n}, \ldots, \mathbf{z}_{m}{ }^{T} \mathbf{n}\right) \cdot \mathbf{f}_{l}=-\mathbf{J}_{n m} \mathbf{f}_{l} .
\end{aligned}
$$

From Eq. (15) to Eq. (18), the internal forces $\mathbf{f}_{n}$ and $\mathbf{f}_{I}$ are given by the following equations.

$$
\begin{aligned}
& \mathbf{f}_{n}=\left(\mathbf{J}_{n m} \mathbf{J}_{c m}{ }^{-1}\right) \mathbf{f}_{c} \\
& \mathbf{f}_{l}=-\mathbf{J}_{c m}{ }^{-1} \mathbf{f}_{c}
\end{aligned}
$$

\subsection{Static force analysis for the redundant PLM}

In this section, the static forces for the redundant 3D4M PLM, as shown in Fig. 2 (b), are derived. Instead of the inverse matrix in Eq. (15), a generalized inverse matrix is applied to the calculation of the static force equation.

$$
\begin{aligned}
& \mathbf{f}_{c}=\left(\mathbf{J}_{c e 43^{T}}\right)^{+} \mathbf{f}_{e}+\left(\mathbf{I}-\left(\mathbf{J}_{c e 43}{ }^{T}\right)^{+} \mathbf{J}_{c e 43}{ }^{T}\right) \mathbf{k} \\
& \mathbf{J}_{c e 43}=\mathbf{J}_{c 4}{ }^{-1} \mathbf{J}_{e 43}
\end{aligned}
$$


where ${ }^{+}$implies the pseudo inverse of a matrix, and $\mathbf{k}$ is a $1 \times 4$ arbitrary vector. In the case of the 3D4M PLM, the left side of (20) has 4 degrees of freedom. On the right side of (20), the 1 st term has 3 degrees of freedom, which means the 2 nd term will have 1 degree of freedom. The null space projection matrix $\left[\mathbf{I}-\left(\mathbf{J}_{c e 43^{T}}\right)^{+} \mathbf{J}_{c e 43^{T}}\right]$ is $4 \times 4$, but its rank is just one. Therefore, the dimension of the 2 nd term on the right side of (20) is reduced to

$$
\begin{aligned}
& \left(\mathbf{I}-\left(\mathbf{J}_{c e 43}{ }^{T}\right)^{+} \mathbf{J}_{c e 43}{ }^{T}\right) \mathbf{k}=\mathbf{J}_{c e 43}{ }^{T} \mathbf{J}_{i}{ }^{-1} \mathbf{f}_{i} \\
& \mathbf{J}_{i}=-\left[\begin{array}{cccc}
\mathbf{z}_{1} & \mathbf{z}_{2} & \mathbf{0} & \mathbf{0} \\
\mathbf{0} & \mathbf{0} & \mathbf{z}_{3} & \mathbf{z}_{4}
\end{array}\right] \\
& \mathbf{f}_{i}=\left[\begin{array}{l}
\mathbf{f}_{i R} \\
\mathbf{f}_{i L}
\end{array}\right]=f_{i}[\cos \theta, \sin \theta,-\cos \theta,-\sin \theta]^{T}
\end{aligned}
$$

The dimension is reduced to one by the independent variable $f_{i}$ in (21). In its physical sense, $f_{i}$ corresponds to the quantity of internal force acting on the end plate.

In Eq. (21), $\mathbf{f}_{i L}$ and $\mathbf{f}_{i R}$ are the internal forces which act upon the left and right ends of the end plate, respectively. For purposes of convenience, they are combined together as the vector $\mathbf{f}_{i}$. The direction of internal forces $\mathbf{f}_{i L}$ and $\mathbf{f}_{i R}$ coincides with the direction of the end plate, whose angle is defined by the rotation angle $\theta$ of the end effecter. The values $\mathbf{f}_{i L}$ and $\mathbf{f}_{i R}$ have the same quantity $f_{i}$, but have opposite directions. The one dimensional internal force that acts along the end plate is explicitly controlled by Eq. (21).

From Eq. (20) and Eq. (21), the generative forces of the actuators $\mathbf{f}_{c}$ are calculated from the external force of the end effecter $\mathbf{f}_{e}$ and the internal force of the end plate $\mathbf{f}_{i}$ as

$$
\mathbf{f}_{c}=\left(\mathbf{J}_{c e 43}{ }^{T}\right)^{+} \mathbf{f}_{e}+\mathbf{J}_{c e 43}{ }^{T} \mathbf{J}_{i}{ }^{-1} \mathbf{f}_{i} .
$$

The internal force of the end plate exerts tensile or compressive stress on the end plate, which helps to diminish joint backlash and increase mechanical rigidity (Adli et al., 1991).

\subsection{Numerical simulation of the static force analysis}

Numerical simulation software of the static force analysis for the 3D4M PLM has been developed. The external force $\mathbf{f}_{e}$, the internal force $\mathbf{f}_{i}$, the generative force of the actuator $\mathbf{f}_{c}$, the constraint force $\mathbf{f}_{n}$ and the tensile force of the link $\mathbf{f}_{l}$ are calculated for an arbitrary position and orientation of the PLM. Examples of static force simulations for the 3D4M PLM are shown in Figs. 8 (a) and (b).

Unit external force $\mathbf{f}_{e}$ is acting at the central position of the end plate. Figure 8 (a) shows the case in which the internal force of the end plate $\mathbf{f}_{i}$ is zero. Representations of the generative force of the actuator $\mathbf{f}_{c}$, the constraint force $\mathbf{f}_{n}$ and the tensile force of the link $\mathbf{f}_{L}$ are superimposed on the link mechanism as a solid line with the symbol * at the tip of the vector. Figure $8(\mathrm{~b})$ shows the case in which the unit internal force of the end plate $\mathbf{f}_{i}$ was applied. In Fig. 8 (b), the internal force $\mathbf{f}_{i}$ and additional forces caused by the $\mathbf{f}_{i}$ at each joint are shown by broken lines. Representations of the generative force of the actuator $\mathbf{f}_{c}$, the constraint force $\mathbf{f}_{n}$ and the tensile force of the link $\mathbf{f}_{L}$ are also superimposed on the link mechanism as a solid line with the symbol * at the tip of the vector. These forces include elements of the internal force $\mathbf{f}_{i}$. The situation in Fig. 8 (b) indicates how compressive forces are exerted upon the end plate. 


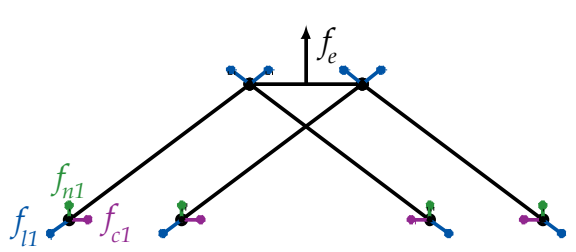

(a) without internal force

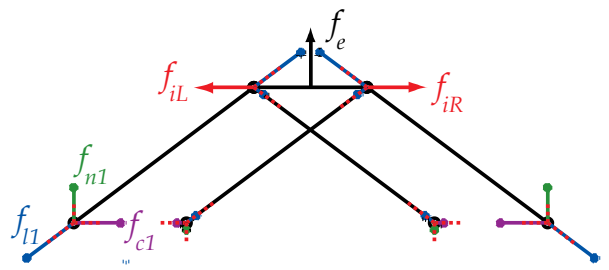

(b) with internal force

Fig. 8. Numerical simulations about static forces

\section{Dynamics of the parallel link mechanism}

\subsection{Equation of motion of the 3D4M PLM}

The positions and orientation of the end plate $\mathbf{p}=[x, y, \theta]^{T}$ and the generative forces and torque at the end effecter $\mathbf{f}_{e}=\left[f_{x}, f_{y}, \tau_{\theta}\right]^{T}$ are considered as generalized positions and generalized forces, respectively. In this formulation, gravity is assumed to be affected by the negative direction of the $y$-axis. The equation of motion is derived by applying Lagrange's equation.

In particular, if the length, mass and moment of inertia of each link is designed with the same value, the equation of motion of the PLM becomes a simple formula, as follows:

$$
\begin{aligned}
& {\left[\begin{array}{l}
f_{x} \\
f_{y} \\
\tau_{\theta}
\end{array}\right]=\left[\begin{array}{ccc}
m_{x x} & 0 & 0 \\
0 & m_{y y}(\mathbf{q}) & m_{y \theta}(\mathbf{q}) \\
0 & m_{y \theta}(\mathbf{q}) & m_{\theta \theta}(\mathbf{q})
\end{array}\right] \ddot{\mathbf{p}}+\left[\begin{array}{c}
0 \\
h_{y}(\mathbf{q}, \dot{\mathbf{q}}) \\
h_{\theta}(\mathbf{q}, \dot{\mathbf{q}})
\end{array}\right]+\left[\begin{array}{c}
0 \\
g_{y} \\
0
\end{array}\right]} \\
& \mathbf{q}=[y, \theta]^{T}
\end{aligned}
$$

Equation (23) represents that the dynamics of the $\mathbf{x}$ direction of the 3D4M PLM has both decoupled and constant inertia characteristics. The dynamics of the $y$ and $\theta$ directions are also decoupled in the $x$ direction. The constant of gravitational force affects only the $y$ direction.

\subsection{Deriving the equation of motion using Mathematica}

Equation (23), the equation of motion of the 3D4M PLM, is derived using Mathematica. The equation of motion is derived by the Lagrange formulation as following steps.

Step 1. Positions (and orientations) of the center of gravity (c.o.g) of mechanical elements rods, end plate and actuator, - are defined as function of the generalized positions, $x(t), y(t)$ and $\theta(t)$.

Step 2. Velocities (and angular velocities) of the c.o.g. of the mechanical elements are derived as the time derivative of the positions (and orientations) of the c.o.g.

Step 3. Kinetic energies and potential energies of the mechanical elements are calculated.

Step 4. The Lagrangian $L$ is derived as the difference between the total kinetic energy $K$ and the total potential energy $U$ of the mechanics.

$$
L=K-U
$$

Step 5. Lagurange's equation of motion is derived as 


$$
\frac{d}{d t}\left(\frac{\partial L}{\partial \dot{q}_{i}}\right)-\frac{\partial L}{\partial q_{i}}=F_{i} .
$$

Where, $q_{i}$ and $F_{i}$ are generalized position and generalized force, respectively.

\subsubsection{Step 1 - positions and orientations of the mechanical elements}

The rod of the 3D4M PLM is designed as an even elongate bar. The c.o.g. of the rod is at the center of the bar. Positions of the c.o.g. of the rods are defined as follows:

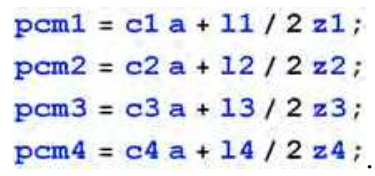

Orientations of the rods are alreday defined as (M. 7). Position and orientation of the end plate are given as (M. 1). Positions of the actuatos are already defined as (M. 5). Positions and orientations of the mechanical elements are defined as functions of the generalised positions, $x(t), y(t)$ and $\theta(t)$.

\subsubsection{Step 2 - velocities and angular velocities of the mechanical elements}

Velocities of the rods are given as time derivative of (M. 16) as follows:

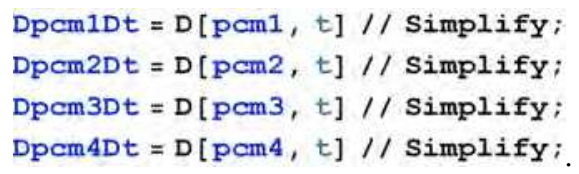

Angular velocities of the rods are given as time derivative of (M. 7) as follows:

$$
\begin{aligned}
& \mathrm{D} \varphi 1 \mathrm{Dt}=\mathrm{D}[\varphi 1, t] / / \text { Simplify; } \\
& \mathrm{D} \varphi 2 \mathrm{Dt}=\mathrm{D}[\varphi 2, t] / / \text { Simplify; } \\
& \mathrm{D} \varphi 3 \mathrm{D} t=\mathrm{D}[\varphi 3, t] / / \text { Simplify; } \\
& \mathrm{D} \varphi 4 \mathrm{Dt}=\mathrm{D}[\varphi 4, t] / / \text { Simplify; }
\end{aligned}
$$

Velocities of the actuators are given as time derivative of (M. 6) as follows:

$$
\begin{aligned}
& \text { Dc1Dt }=D[c 1, t] ; \\
& D c 2 D t=D[c 2, t] ; \\
& D c 3 D t=D[c 3, t] ; \\
& D c 4 D t=D[c 4, t] ;
\end{aligned}
$$

Velocity and angular velocity of the end plate are given as time derivative of (M. 1) as follows:

$$
\begin{aligned}
& \text { DpDt }=D[p, t] ; \\
& \text { DeDt }=D[\theta[t], t] ; .
\end{aligned}
$$

\subsubsection{Step 3 - kinetic energies and potential energies of the mechanical elements} Kinetic energy $\mathrm{K} i$ of each rod, and $K t$ of the end plate are defined as follows: 


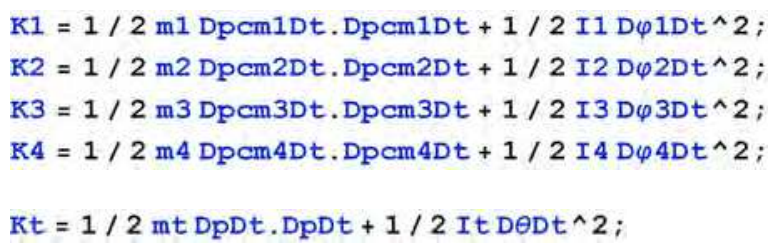

Mass and moment of inertia of the $i$ th rod are defined as $m i$ and $I i$, respectively. Mass and moment of inertia of the end plate are expressed as $m t$ and $I t$, respectively.

Sum of kinetic energies of the actuators are expressed as

$\mathrm{Km}=1 / 2 \operatorname{mm} 1 \mathrm{Dc} 1 \mathrm{Dt} t^{\wedge} 2+1 / 2 \operatorname{mm} 2 \mathrm{Dc} 2 \mathrm{Dt} t^{\wedge} 2+1 / 2 \operatorname{mm} 3 \mathrm{Dc} 3 \mathrm{Dt} t^{\wedge} 2+$

$1 / 2 \operatorname{mm} 4 \operatorname{Dc} 4 \mathrm{Dt}^{\wedge} 2$;

Mass of the $i$ th actuator is expressed as mmi.

Potential energies of rods and end plate are expressed as follows:

$$
\begin{aligned}
& \mathrm{U} 1=\mathrm{m} 1 \mathrm{~g} \mathrm{p} \mathrm{pm} 1 \llbracket 2 \rrbracket ; \\
& \mathrm{U} 2=\mathrm{m} 2 \mathrm{~g} \text { p cm2 } \llbracket 2 \rrbracket \text {; } \\
& \mathrm{U} 3=\mathrm{m} 3 \mathrm{~g} \text { pcm } 3 \llbracket 2 \rrbracket ; \\
& \mathrm{U} 4=\mathrm{m} 4 \mathrm{~g} \mathrm{pcm} 4 \llbracket 2 \rrbracket ; \\
& \mathrm{Ut}=\mathrm{mtg} \mathrm{p} \llbracket 2 \rrbracket ;
\end{aligned}
$$

\subsubsection{Step 4 - The Lagrangian of the mechanism}

The Lagrangian $L$ is derived as the difference between the total kinetic energy $K$ of (M. 21) (M. 23) and the total potential energy $U$ of (M. 24) as follows:

$$
L=K 1+K 2+K 3+K 4+K t+K m-(U 1+U 2+U 3+U 4+U t)
$$

\subsubsection{Step 5 - Lagurange's equation of motion of the mechanism}

Equation of motion of the mechanism is derived by applying Lagrange's equation of Eq. (25) to (M. 25) as follows.

$$
\begin{aligned}
& S 1=D\left[D\left[L, x^{\prime}[t]\right], t\right]-D[L, x[t]] ; \\
& S 2=D\left[D\left[L, y^{\prime}[t]\right], t\right]-D[L, y[t]] ; \\
& S 3=D\left[D\left[L, \theta^{\prime}[t]\right], t\right]-D[L, \theta[t]] ;
\end{aligned}
$$

S1, S2 and S3 are the left parts of Lagrange's equation of Eq. (25).

\subsection{Simplify the dynamic characteristics of the 3D4M}

As shown in Eq. (23), if the length, mass and moment of inertia of each link is designed with the same value, the equation of motion of the 3D4M PLM becomes a simple formula. Here we derive Eq. (23) using symbolic analysis by Mathematica.

Mass and moment of inertia of each mechanical part is descrived as follows.

$$
\begin{aligned}
\text { Ink1st } & =\{\mathrm{mm} 1 \rightarrow \mathrm{mm}, \mathrm{mm} 2 \rightarrow \mathrm{mm}, \mathrm{mm} 3 \rightarrow \mathrm{mm}, \mathrm{mm} 4 \rightarrow \mathrm{mm}, 11 \rightarrow 1, \\
12 & \rightarrow 1,13 \rightarrow 1,14 \rightarrow 1, \mathrm{~m} 1 \rightarrow \mathrm{ml}, \mathrm{m} 2 \rightarrow \mathrm{ml}, \mathrm{m} 3 \rightarrow \mathrm{ml}, \mathrm{m} 4 \rightarrow \mathrm{ml}, \\
I 1 & \rightarrow \mathrm{Il}, \mathrm{I} 2 \rightarrow \mathrm{Il}, \mathrm{I} 3 \rightarrow \mathrm{I} 1, \mathrm{I} 4 \rightarrow \mathrm{Il}\} ;
\end{aligned}
$$


By applying the list (M. 27) to (M. 26), the equation of motion of the 3D4M PLM becomes

$$
\begin{aligned}
& \mathrm{s} 1 \mathrm{~b}=\mathrm{s} 1 / . \text { Inklst } \\
& \mathrm{s} 2 \mathrm{~b}=\mathrm{s} 2 / . \text { Inklst } \\
& \mathrm{s} 3 \mathrm{~b}=\mathrm{s} 3 / . \text { Inklst; }
\end{aligned}
$$

Left side of equation of motion along the direction $x$ becomes as follows:

S1b // Simplify

$$
(4 m]+4 m m+m t) x^{\prime \prime}[t] .
$$

The equation of motion along the direction $\mathrm{x}$ is reduced to

$$
\left(4 m_{l}+4 m_{m}+m_{t}\right) \ddot{x}(t)=f_{x}
$$

Equation (26) indicates that the dynamics of the direction $\mathbf{x}$ of the 3D4M PLM has both decoupled and constant inertia characteristics.

We obtain the gravity terms of the equation of motion as

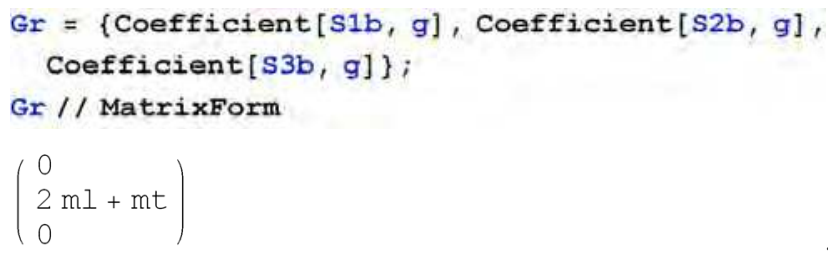

(M. 30) indicates that the constant of gravitational force $g_{y}=\left(2 m_{l}+m_{t}\right) g$ affects only to the direction $y$. Effective mass $m_{y}$ and $m_{\theta}$ of the direction $y$ in Eq. (23) are derived as

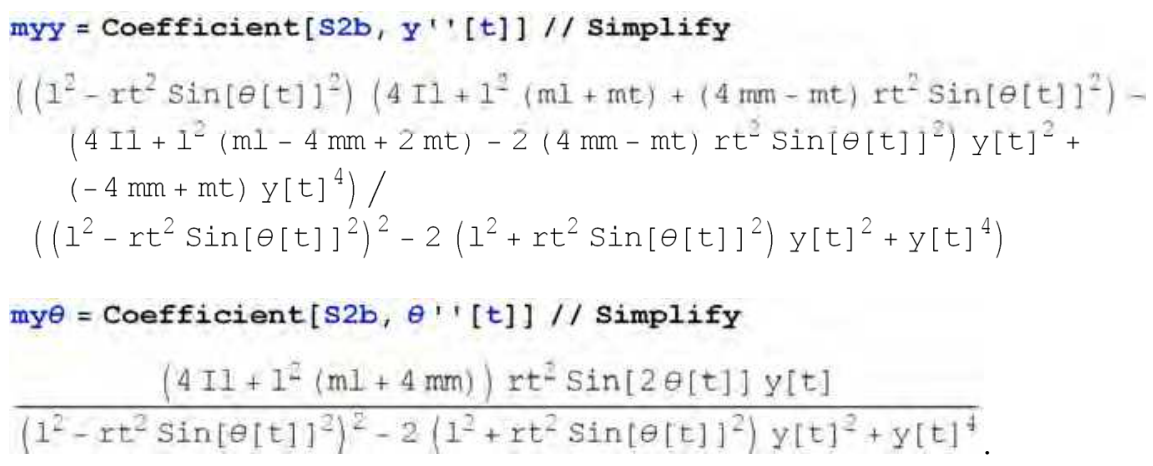

Coeffcients of Coriolis and centrifigual forces of the durection $y$ in Eq. (23) are reduced to

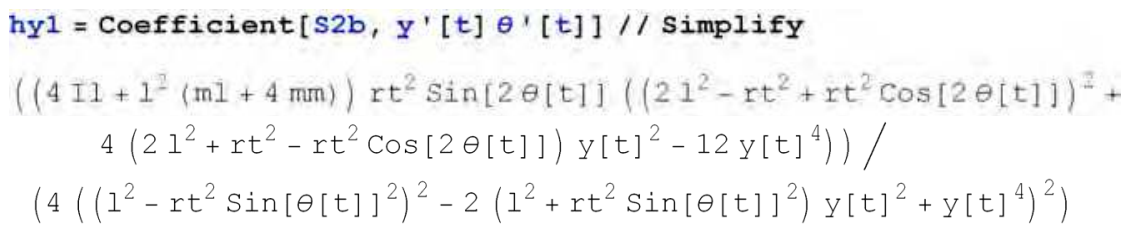




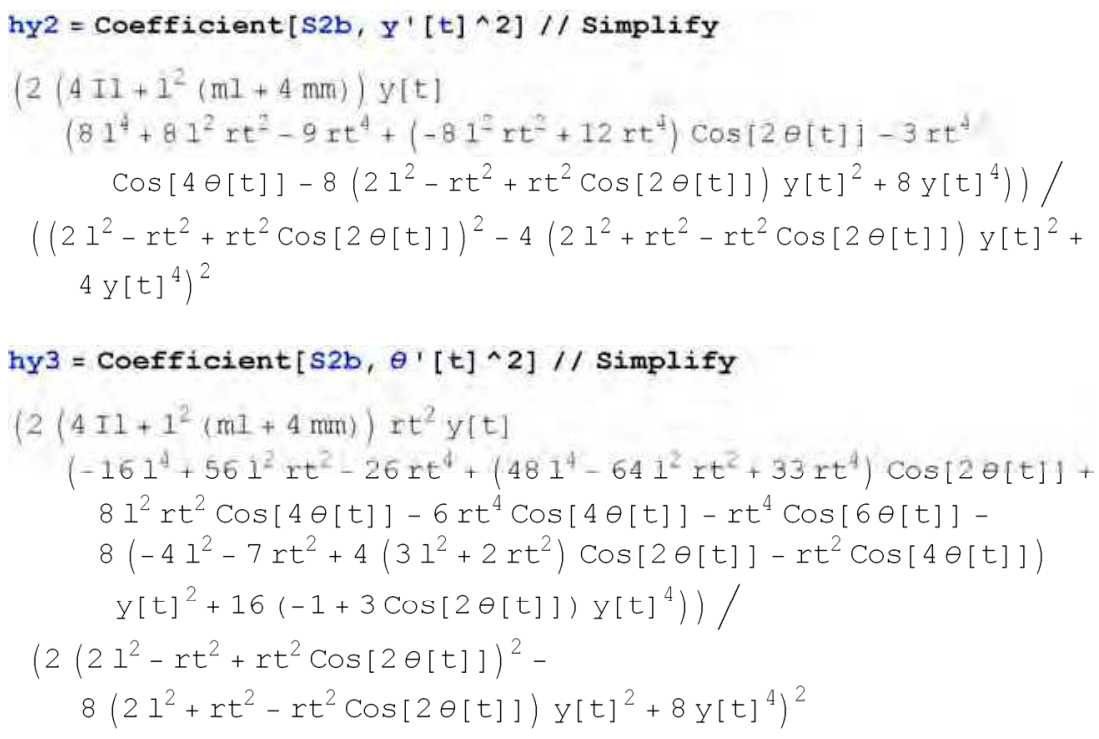

Then, $h_{y}(\mathbf{q}, \dot{\mathbf{q}})$, Coriolis and centrifigual forces of the durection $y$ in Eq. (23), is given as

$$
h_{y}(\mathbf{q}, \dot{\mathbf{q}})=h_{y 1} \dot{y} \dot{\theta}+h_{y 2} \dot{y}^{2}+h_{y 3} \dot{\theta}^{2} .
$$

Effective moment of inertia $m_{\theta \theta}$ and Corioli and centrifigual moment $h_{\theta}$ of the direction $\theta$ are given as the same mannar of (M. 31) - (M. 35).

\section{Numerical simulations of the dynamics}

Numerical simulations of the dynamics of the 3D4M PLM has been tested using Mathematica. Values of the kinematic parameters of the 3D4M PLM are set as Table 1. These values are given from the prototype of the 3D4M PLM (Harada \& Nagase, 2010) as show in Fig. 9. Values of the kinematic parameters are given follows:

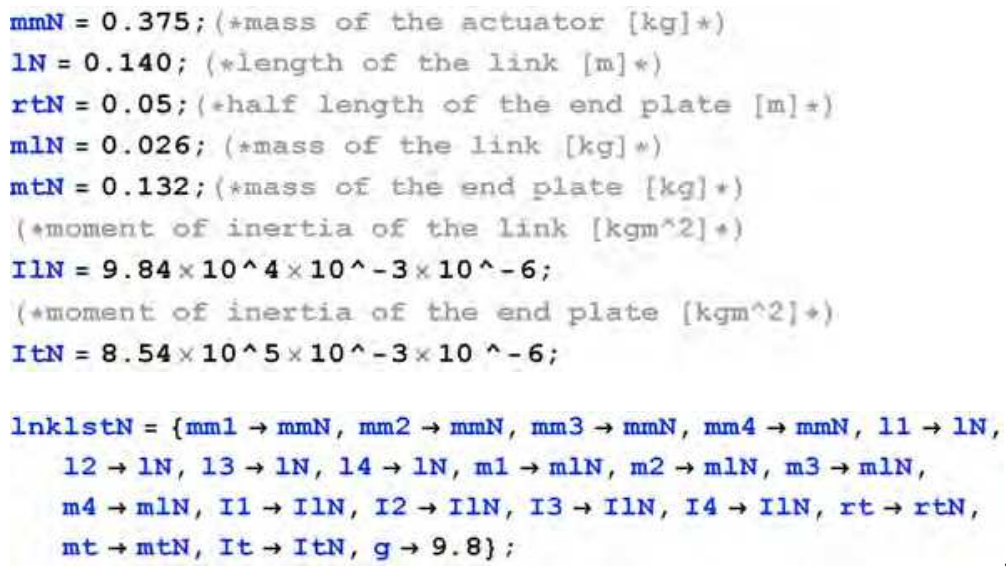




\begin{tabular}{|c|l|c|}
\hline \multirow{3}{*}{ Link } & Length [m] & 0.140 \\
\cline { 2 - 3 } & Mass [kg] & 0.026 \\
\cline { 2 - 3 } & Moment of inertia $\left[\mathrm{kgm}^{2}\right]$ & $9.84 \times 10^{-5}$ \\
\hline \multirow{3}{*}{ End plate } & Length [m] & 0.100 \\
\cline { 2 - 3 } & Mass [kg] & 0.132 \\
\cline { 2 - 3 } & Moment of inertia $\left[\mathrm{kg} \mathrm{m}^{2}\right]$ & $8.54 \times 10^{-4}$ \\
\hline Actuator & Total mass [kg] & 0.375 \\
\hline
\end{tabular}

Table 1. Specifications of the mechanical parts of the prototype
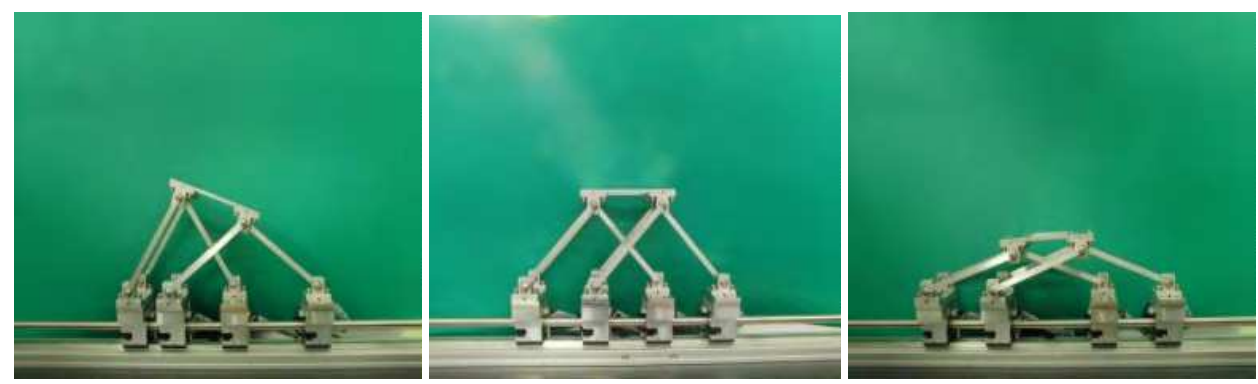

Fig. 9. Front views of the prototype

Equations of motion of Eq. (23) are nonlinear simultaneous ordinary differential equations about $x(t), y(t)$ and $\theta(t)$ with respect to time $t$. They are numerically solved by the operator "NDSolve" in Mathematica.

We show an example of numerical simulation with initial conditions of the generalized positions and velocities are set as Table 2 .

\begin{tabular}{|c|c|c|c|}
\hline \multicolumn{2}{|c|}{ positions } & \multicolumn{2}{c|}{ velocities } \\
\hline$x(0)[\mathrm{m}]$ & 0.0 & $\dot{x}(0)[\mathrm{m} / \mathrm{s}]$ & 0.0 \\
\hline$y(0)[\mathrm{m}]$ & 0.08 & $\dot{y}(0)[\mathrm{m} / \mathrm{s}]$ & 0.0 \\
\hline$\theta(0)[\mathrm{deg}]$ & 20.0 & $\dot{\theta}(0)[\mathrm{rad} / \mathrm{s}]$ & 0.0 \\
\hline
\end{tabular}

Table 2. Initial conditions of the numerical simulation

Constant generalized forces and torque are applied as Table 3.

\begin{tabular}{|c|c|}
\hline \multicolumn{2}{|c|}{ forces and torque } \\
\hline$f_{x}[\mathrm{~N}]$ & 0.2 \\
\hline$f_{y}[\mathrm{~N}]$ & $\left(2 m_{l}+m_{t}\right) g$ \\
\hline$\tau_{\theta}[\mathrm{Nm}]$ & 0.0 \\
\hline
\end{tabular}

Table 3. Generalized forces and torque of the numerical simulation 1

Constant force $f_{y}=\left(2 m_{l}+m_{t}\right) g$ compensates the gravitational effect along the direction $y$ as shown in (M. 30).

Responses of the generalized positions with the conditions of Table $2 \& 3$ from time $t=0$ [s] to $t=2.0$ [s] are numerically calculated by Mathematica as follows: 


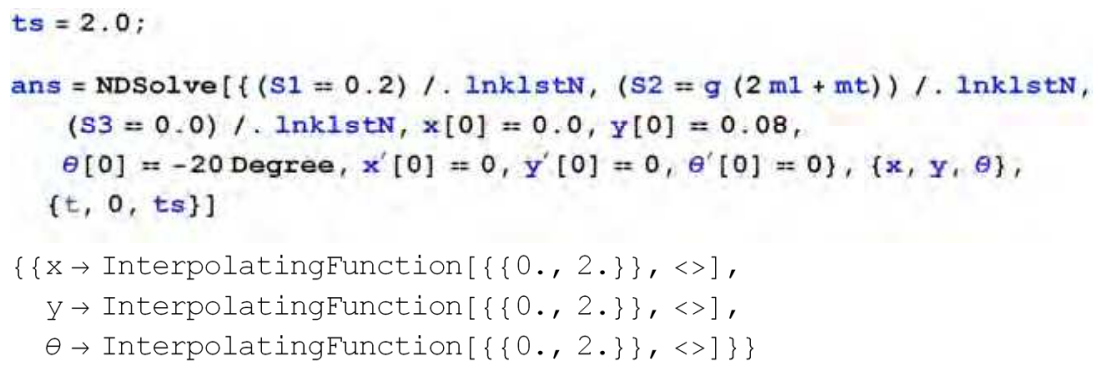

The results are graphically shown by using Mathematica with the following codes.

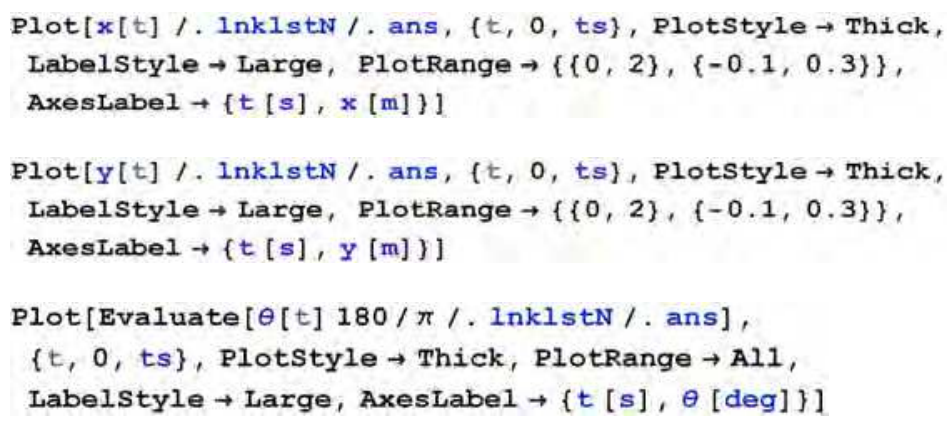

Plot [Evaluate $[\theta[t] 180 / \pi /$. InklstN /. ans],

Outputs of (M. 39) and (M. 41) are shown as Fig. 10 (a), (b) and (c), respectively.

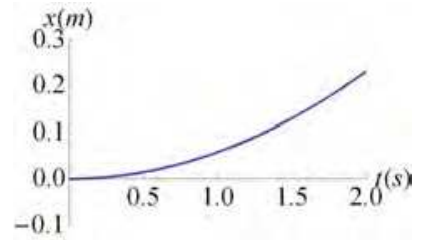

(a) $x$

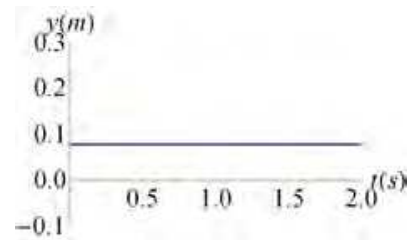

(b) $y$

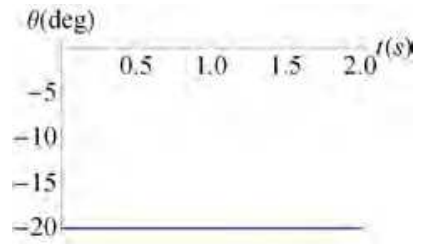

(c) $\theta$

Fig. 10. Results of the numerical simulation 1 (generalized positions)

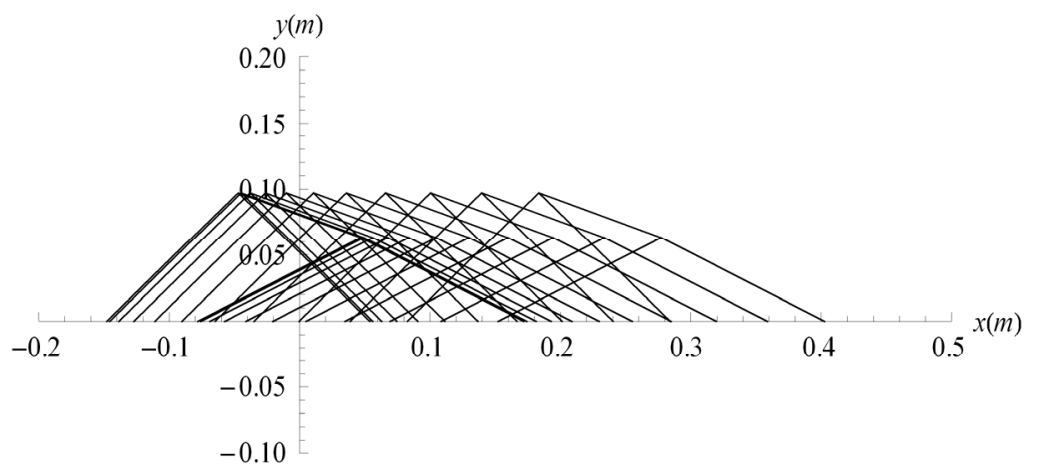

Fig. 11. Results of the numerical simulation 1 (skeletons) 
Motions of the mechanism are graphically shown by the following code of Mathematica.

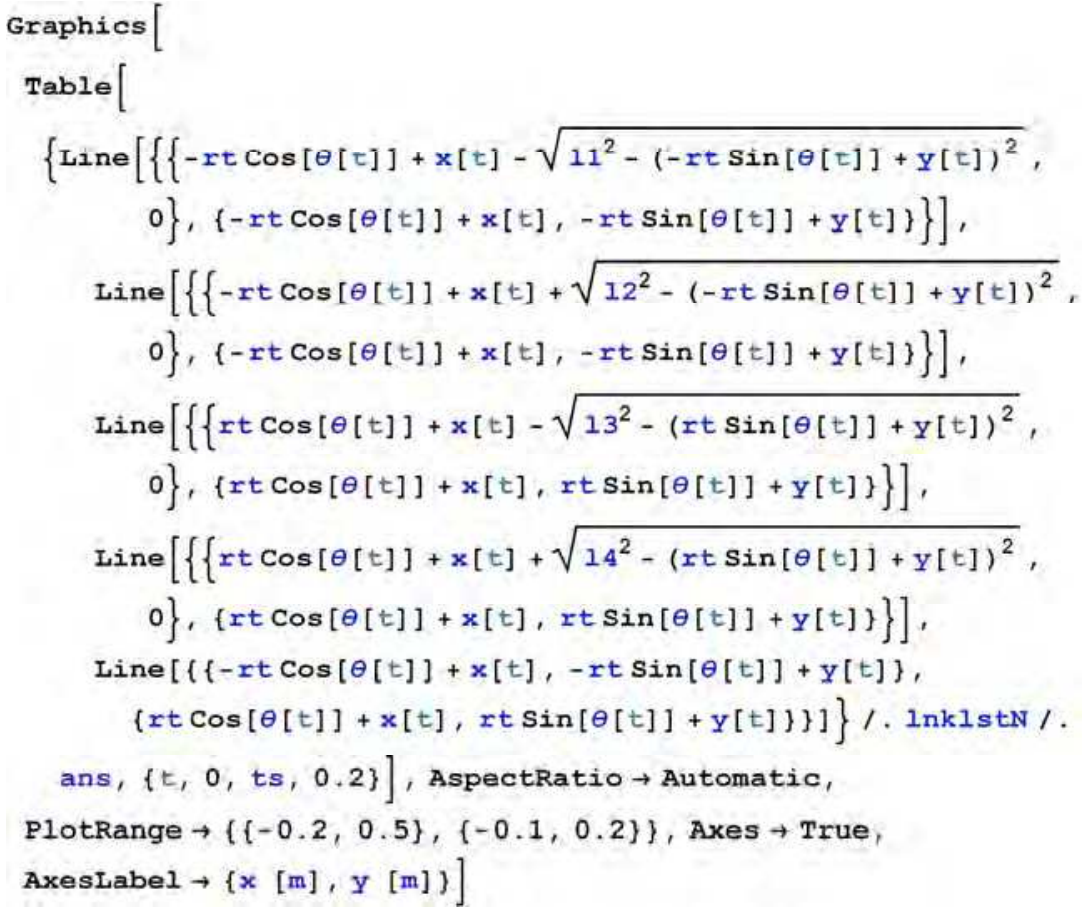

Output of (M. 41) is shown as Fig. 11.

As shown in Fig. 10 and Fig. 11, the 3D4M PLM generated a constant motion of acceleration in the direction $x$ while maintaining its initial configuration. The characteristiocs of the constant and decoupled dynamics of the 3D4M PLM along the direction $x$ has been confirmed by this simulation.

We show another example of numerical simulation with initial conditions of the generalized positions and velocities are set same as Table 2, but other types of constant generalized forces and torque are applied as Table 4.

\begin{tabular}{|c|c|}
\hline \multicolumn{2}{|c|}{ forces and torque } \\
\hline$f_{x}[\mathrm{~N}]$ & 0.0 \\
\hline$f_{y}[\mathrm{~N}]$ & $\left(2 m_{l}+m_{t}\right) g$ \\
\hline$\tau_{\theta}[\mathrm{Nm}]$ & 0.005 \\
\hline
\end{tabular}

Table 4. Generalized forces and torque of the numerical simulation 2

Code for solving the nonlinear simultaneous ordinary differential equations is given as

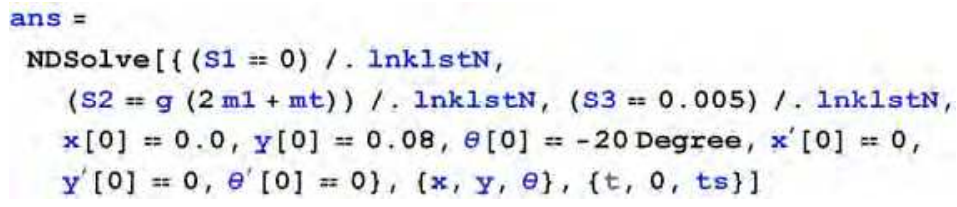


Results of the simulation are shown in Fig. 12 and Fig. 13.

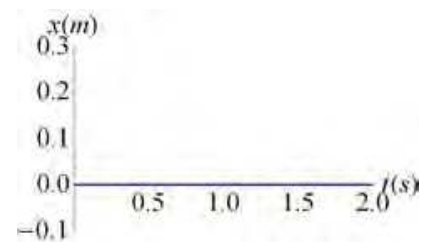

(a) $x$

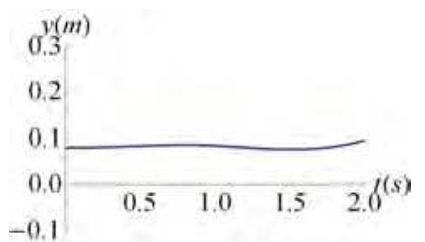

(b) $y$

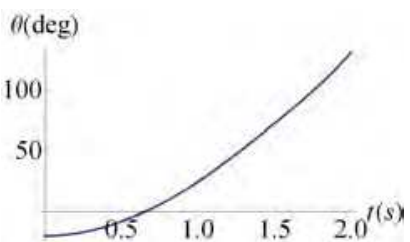

(c) $\theta$

Fig. 12. Results of the numerical simulation 2 (generalized positions)

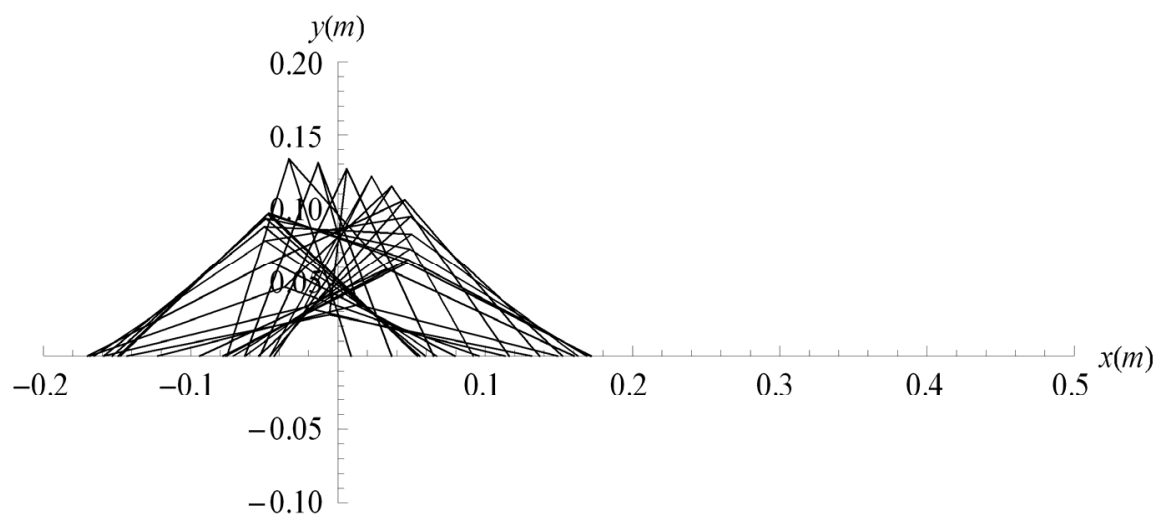

Fig. 13. Results of the numerical simulation 2 (skeletons)

As shown in Fig 12 and Fig. 13, the 3D4M PLM generated a constant motion of acceleration in the $\theta$ direction while maintaining its initial horisontal $(x)$ position. As shown if Fig. 12 (b), position of the direction $y$ slightly moves because of the dynamical coupling between the directions $y$ and $\theta$.

\section{Conclusion}

A novel redundantly actuated planar parallel link mechanism using multi drive linear motors has been proposed. It expands the range of motion, while retaining the advantages of rigid mechanism and precise positioning. The kinetostatics and dynamics characteristics of the mechanism have been analyzed using symbolic mathematical analysis and numerical simulations.

1. Kinematic equations, forward kinematics and derivative kinematics of the mechanism have been derived. The derived equations have been symbolically programmed using Mathematica.

2. Singularity and static forces of the mechanism have been analyzed using Mathematica. The conditions of the singularity have been symbolically solved using Mathematica.

3. The equations of motion of the mechanism have been symbolically derived using Mathematica by Lagrange's formulation. 
4. If the length, mass and moment of inertia of each link were designed with the same value, the equations of motion of the mechanism have been showed the decoupled and constant inertia characteristics in the horizontal direction.

5. Examples have been introduced for solving the equations of motion numerically using Mathematica. The characteristics of the decoupled and constant inertia of the mechanism has been confirmed by the simulations.

\section{Acknowledgment}

This work was supported in part by the Electro-Mechanic Technology Advancing Foundation and Mazak Foundation, Japan.

\section{References}

Adli, M. A.; Nagai K.; Miyataka, K. \& Hanafusa, H. (1991). Analysis of Internal Force Effect in Parallel Manipulators, Transactions of the Society of Instrument and Control Engineers, Vo. 27, No. 11, pp. 1266-1273, ISSN 0453-4654

Arai, T.; Stoughton, R.; Homma, K.; Adachi, H.; Nakamura, T. \& Nakashima, K. (1991). Development of a parallel link manipulator," Proceedings of 5th International Conference on Advanced Robotics, Vol. 1, pp. 839-844, ISBN 0-7803-0078-5, Pisa , Italy, 19-22 Jun 1991

Chiu, Y. J. \& Perg, M. H. (2003). Self-calibration of a general hexapod manipulator using cylindrical constraints, International Journal of Machine Tools $\mathcal{E}$ Manufacture, Vol. 43, Issue 10, pp. 1051-1066, ISSN 0890-6955

Gosselin, C. \& Angeles, J. (1990). Singularity analysis of closed-loop kinematic chains," IEEE Transactions on Robotics and Automation, Vol. 6, No.3, pp. 281-290, ISSN 1042-296X

Harada, T. \& Nagase M (2009). Configurations and Mathematical Models of Parallel Link Mechanisms Using Multi Drive Linear Motors, Proceedings of the 2009 IEEE/RSJ International Conference on Intelligent Robots and Systems, pp. 1974-1979, ISBN 978-14244-3803-7, St. Louis, MO, USA, 10-15 Oct. 2009

Harada, T. \& Nagase M (2010). Impedance Control of a Redundantly Actuated 3-DOF Planar Parallel Link Mechanism Using Direct Drive Linear Motors, Proceedings of the 2010 IEEE International Conference on Robotics and Biomimetics, pp. 501-506, ISBN 978-1-4244-9317-3, Tianjin, China, 14-18 Dec. 2010

Hogan, N. (1985). Impedance Control: An Approach to Manipulation: Part I-III, Transactions of the ASME, Journal of Dynamic Systems, Measurement, and Control, Vol. 107, No. 1, pp. 1-24, ISSN 0022-0434

Honegger, M.; Codourey, A. \& Burdet E. (1997). Adaptive Control of the Hexaglide, A 6 DOF Parallel Manipulator, Proceedings of the 1997 IEEE International Conference on Robotics and Automations, pp. 21-28, ISBN 0-7803-3612-7, Albuquerque, NM , USA, 20-25 Apr 1997

In, W.; Lee, S.; Jeong, J. I. \& Kim, J. (2008). Design of a Planar-Type High Speed Parallel Mechanism Positioning Platform with the Capability of 180 Degrees Orientation, CIRP Annals - Manufacturing Technology, Vol. 58, Issue 1, pp. 421-424, ISSN 00078056

Kim, J.; Cho, Y. M.; Park, F. C. \& Lee, J. M. (2003). Design of a Parallel Mechanism Platform for Simulating Six Degrees-of-freedom General Motion Including Continuous 360- 
degree Spin, CIRP Annals - Manufacturing Technology, Vol. 52, Issue 1, pp. 347-350, ISSN 0007-8056

Leong, J. I.; Kang, D.; Cho, Y. M \& Kim J. (2004), Kinematic Calibration for Redundantly Actuated Parallel Mechanisms, Transactions of the ASME, Journal of Mechanical Design, Vol. 126, Issue 2, pp. 307-319, ISSN 1050-0472

Liu, X. J.; Wang, J. \& Pritschow, G. (2004). A New Family of Spatial 3-DOF Fully Parallel Manipulators with High Rotational Capability, Mechanism and Machine Theory, pp. 475-494, ISSN 0094-114X

Liu, X. J.; Guan, L. \& Wang J. (2007), Kinematics and Closed Optimal Design of a Kind of PRRRP Parallel Manipulator, Transactions of the ASME, Journal of Mechanical Design, Vol. 129, Issue 5, pp. 558-563, ISSN 1050-0472

Marquet, F.; Krut, S.; Company, O. \& Pierrot, F. (2001). ARCHI: A New Redundant Parallel Mechanism - Modeling, Control and First Results, Proceedings of the 2001 IEEE/RSJ International Conference on Intelligent Robots and Systems, pp. 183-188, ISBN 0-78036612-3, Maui, HI, USA, 29 October - 03 November 2001

Merlet, J. P. (1989). Singular configurations of parallel manipulators and grassmann geometry, The International Journal of Robotics Research, Vol. 8, No. 5, pp. 45-56, ISSN 0278-3649

Merlet, J. P. (1996). Direct kinematics of planar parallel manipulators, Proceedings of 1996 IEEE International Conference on Robotics and Automation, Vol. 4, pp. 3744 - 3749, ISBN 0-7803-2988-0, Minneapolis, MN , USA, 22-28 Apr 1996

Merlet, J. P. (2006). Parallel Robots (Second Edition), Springer, ISBN 978-1-4020-4132-7, Netherland

Milutinovic, D. S.; Glavonjic, M.; Kvrgic, V. \& Zivanovic S. (2005). A New 3-DOF Spatial Parallel Mechanism for Milling Machines with Long X Travel, CIRP Annals Manufacturing Technology, Vol. 54, Issue 1, pp. 345-348. ISSN 0007-8056

Oiwa, T. (1997). New coordinate measuring machine featuring a parallel mechanism, International journal of the Japan Society for Precision Engineering, Vol. 31, No. 3, pp. 232-233, ISSN 0916-782X

Stewart, D. (1966). A platform with six degrees of freedom, Proceedings of the Institution of Mechanical Engineers, Vol. 180, No. 15, pp. 371-386, ISSN 0020-3483

Wang, J. \& Liu, X. J. (2008). Parallel Robots: Recent Advances in Research and Application, Nova Science Pub Inc., ISBN 978-1-60456-859-2, New York

Wang, J.; Wu, J.; Li, T. \& Liu, X. (2008). Workspace and Singularity Analysis of a 3-DOF Planar Parallel Manipulator with Actuation Redundancy, Robotica, Vol. 27, pp. 5157, ISSN 0263-5747

Weck, M. (2002). Parallel kinematic machine tools-current state and future potentials, CIRP Annals - Manufacturing Technology, Vol. 51, Issue 2, pp. 671-683, ISSN 0007-8056

Yoshikawa, T. (1985). Manipulability of Robotic Mechanisms, The International Journal of Robotic Research, Vol. 4, No. 2, pp. 3-9, ISSN 0278-3649

Zhang, J. (2008), A Family of Parallel Manipulators with Mobile Platform Rotating Continuously, in Parallel Robotics: Advances in Research and Application, J. Wang, and X. J. Liu, Ed., New York: Nova Science Publishers, Inc., 2008, pp. 67-80.

Zhuang, H. \& Liu, L. (1998). Determination of number of independent parameters for the self-calibration of parallel-link mechanisms, Proceedings of ASME, MED-vol.8, pp. 699-703, 1998. 


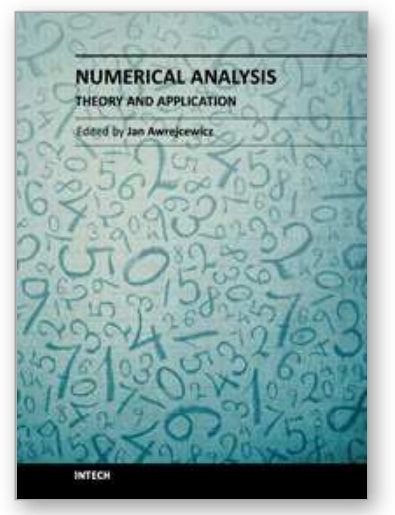

\author{
Numerical Analysis - Theory and Application \\ Edited by Prof. Jan Awrejcewicz
}

ISBN 978-953-307-389-7

Hard cover, 626 pages

Publisher InTech

Published online 09, September, 2011

Published in print edition September, 2011

Numerical Analysis â€“ Theory and Application is an edited book divided into two parts: Part I devoted to Theory, and Part II dealing with Application. The presented book is focused on introducing theoretical approaches of numerical analysis as well as applications of various numerical methods to either study or solving numerous theoretical and engineering problems. Since a large number of pure theoretical research is proposed as well as a large amount of applications oriented numerical simulation results are given, the book can be useful for both theoretical and applied research aimed on numerical simulations. In addition, in many cases the presented approaches can be applied directly either by theoreticians or engineers.

\title{
How to reference
}

In order to correctly reference this scholarly work, feel free to copy and paste the following:

Takashi Harada (2011). Kinetostatics and Dynamics of Redundantly Actuated Planar Parallel Link Mechanisms, Numerical Analysis - Theory and Application, Prof. Jan Awrejcewicz (Ed.), ISBN: 978-953-307389-7, InTech, Available from: http://www.intechopen.com/books/numerical-analysis-theory-andapplication/kinetostatics-and-dynamics-of-redundantly-actuated-planar-parallel-link-mechanisms

\section{INTECH}

open science | open minds

\section{InTech Europe}

University Campus STeP Ri Slavka Krautzeka 83/A 51000 Rijeka, Croatia Phone: +385 (51) 770447

Fax: +385 (51) 686166 www.intechopen.com

\section{InTech China}

Unit 405, Office Block, Hotel Equatorial Shanghai No.65, Yan An Road (West), Shanghai, 200040, China 中国上海市延安西路65号上海国际贵都大饭店办公楼 405 单元 Phone: +86-21-62489820

Fax: $+86-21-62489821$ 
(C) 2011 The Author(s). Licensee IntechOpen. This chapter is distributed under the terms of the Creative Commons Attribution-NonCommercialShareAlike-3.0 License, which permits use, distribution and reproduction for non-commercial purposes, provided the original is properly cited and derivative works building on this content are distributed under the same license. 